\title{
THE KURAMOTO MODEL SUBJECT TO A FLUCTUATING ENVIRONMENT: APPLICATION TO BRAINWAVE DYNAMICS
}

\author{
A. C. HALE*, T. HANSARD, L. W. SHEPPARD, P. V. E. McCLINTOCK \\ and A. STEFANOVSKA \\ Department of Physics, \\ Lancaster University, Lancaster, LA1 $4 Y B$, UK \\ *a.c.hale@lancaster.ac.uk \\ Received (received date) \\ Revised (revised date)
}

\begin{abstract}
We consider the phase dynamics of an ensemble of Kuramoto oscillators whose eigenfrequencies are perturbed to model the openness of living systems, and we show that it exhibits time-localized epochs of synchrony. A new quantitative measure is used to show that the model compares well with electroencephalography (EEG) data recorded from a healthy awake human.
\end{abstract}

Keywords: nonlinear dynamics; noise; coupled oscillators; brain models; synchronization; time series analysis.

\section{Introduction}

Globally-coupled nonlinear oscillators are found in a diverse range of selfsynchronizing ensembles in physics, chemistry, communications and living systems. Often, they are open, dissipative, and function far from thermodynamic equilibrium. Systems of this kind frequently exhibit order, e.g. through spontaneous synchronization $[1,2]$. In what follows, we model a class of open, self-synchronized, oscillatory systems relevant to e.g. the collective behavior of neurons in the brain.

Brain models may be grouped into (a) neuron models [3], and (b) macroscopic field models [4-6]. Such models may include stochastic forcing terms [7] to account for the unpredictability of neuronal firing dynamics brought about by the brain's complex connectivity. We will make use of the Kuramoto model, a class (b) model increasingly being applied in neuroscience [8-10], for which the dynamics are based solely upon phase because any amplitude dynamics is assumed negligible. Here we emulate macroscopic brainwave dynamics with a modified form of the model. We use each Kuramoto oscillator to represent a cluster of neurons (i.e. a macrocolumn) that mutually interact to yield coherent oscillatory behavior. A potential difficulty is that Kuramoto models force all members of the ensemble to behave in a synchronized manner for sufficiently strong coupling, so that there is a danger of the model becoming too highly synchronized to represent a healthy human brain. We 
therefore make use of an "open phase dynamics" approach to model macroscopic brain activity as seen in electroencephalography (EEG) signals, in particular to try to encompass their intermittent synchronization. Synchrony is seen as being disrupted by information/matter flow [11]. This results in alternating epochs of synchronized and de-synchronized states caused by the openness to environmental influences, while feedback mechanisms inherent in the Kuramoto system allow the system to self-regulate and maintain stability.

In Sec. 2 we introduce the Kuramoto model and propose a method of generalizing it to describe open systems where the influence of the environment cannot be neglected. We summarize in Sec. 3 a recently-introduced method for quantifying the degree of synchronization in an ensemble of coupled oscillators in terms of a quantity $\kappa$, and we apply these ideas to a human EEG signal in Sec. 4. In Sec. 5 we apply the generalized Kuramoto system to provide a minimalistic model of macroscopic brain function using phase dynamics. Openness is simulated, not by smooth time-varying functions (as in classic non-autonomous oscillatory systems), but instead by discrete discontinuous change in the system's state, representing its response to the environment. These changes are effected by perturbing some of the oscillators away from their natural eigenfrequencies. We show that the result is episodic synchronization. Estimating the time-scales of this intermittency from the EEG data, and using $\kappa$ as a measure, we show that the model can provide a satisfactory description of the episodic character of EEG synchronization. In Sec. 6 we summarize the results and draw conclusions.

\section{An open phase-dynamics model}

In the simplest case, a self-synchronizing system of oscillators is closed. If their dynamics is governed, to a good approximation, by phase dynamics then it may be described by the classic Kuramoto model $[1,2]$ :

$$
\dot{\phi}_{p}(t)=\omega_{p}+\frac{K}{N} \sum_{q=1}^{N} \sin \left(\phi_{q}(t)-\phi_{p}(t)\right), \quad p=1, \ldots, N .
$$

Here $\phi_{p}$ is the phase of the $p^{t h}$ oscillator at time $t$ with eigenfrequency $\omega_{p}$ chosen at random from a unimodal, symmetric probability density $g(\omega)$. Coupling between the $N$ nodes is all-to-all, with strength $K \in \mathbb{R}^{+}$. Initially, at time $t=0$, all the phases are randomly selected from a uniform distribution $[0 \ldots 2 \pi)$. The collective behavior is quantified by the complex order parameter:

$$
r(t)=\Psi(t) e^{i \Phi(t)}=\frac{1}{N} \sum_{q=1}^{N} e^{i \phi_{q}(t)},
$$

where $\Phi(t)$ is the average phase and the mean field amplitude $0<\Psi(t)<1$ is a measure of the system's macroscopic coherence (i.e. synchronization). The nearer $\Psi$ is to unity, the higher the degree of global synchronization; zero denotes the incoherent (de-synchronized) state. 
A natural generalization of this Kuramoto system is to extend it to the open case. This is typically realized by the addition of a noise term to Eq. (1), where statistically-independent equally-distributed noisy forces are applied to each oscillator [2]. The noise term is commonly Gaussianly-distributed with zero mean. Another class of openness can be achieved by adjusting Eq. (1) such that $\omega_{p}$ and/or $K$ become time-dependent in a deterministic manner; for example the coupling term could be $\mathcal{K}(t)=t K / \tau$ leading to complete system-wide synchronization in time $t \approx \tau\left|\omega_{p}^{\max }\right| / K$ (where $\left|\omega_{p}^{\max }\right|$ is the highest eigenfrequency amongst $N$ oscillators and $\tau \in \mathbb{R}^{+}$). In [9], each oscillator was subject to an independent Gaussian white noise and, in addition, the coupling parameter was ramped up in time to simulate a decrease in anæsthetic concentration.

Here we create, instead, a class of open systems where the $p^{\text {th }}$ oscillator in the network has its eigenfrequency perturbed to a new frequency picked from a stochastic distribution. After sufficient time, all oscillators will have their original eigenfrequency perturbed and then later reset; after which the cycle repeats. Conceptually this is similar to considering a set of driven "Huygens' pendulums" (each of approximately the same eigenfrequency) hanging from, and coupled via, a wooden beam; knocking any one or more of the pendulums will represent introducing information (or noise) into the system but, with sufficient time, it will return to its original state. Unless kicked in this way this system can carry no information and is closed.

In general, if a perturbation occurs too frequently, it will tend to completely desynchronize the system: if there is insufficient time for the transients to die away, the system will be in a continuous state of agitation. If the system is sufficiently strongly coupled, and the rate at which oscillators are perturbed is sufficiently slow, then it will tend towards a synchronized state.

The kick to each eigenfrequency represents a flow of information/energy/matter in and out of the system, corresponding to its openness. In this sense, our system represents a discrete, nonautonomous, Kuramoto model with a special timedependent term $\chi_{p}(t)$ which perturbs the eigenfrequency:

$$
\dot{\phi}_{p}(t)=\omega_{p}+\frac{K}{N} \sum_{q=1}^{N} \sin \left(\phi_{q}(t)-\phi_{p}(t)\right)+\chi_{p}(t) .
$$

The perturbation to the eigenfrequency $\chi_{p}(t)$ is chosen at random from a unimodal, symmetric probability density $g(\chi)$; this distribution is distinct from $g(\omega)$. In many biological systems the external environment could have a non-equilibrium probability distribution $g(\chi)$, or there could exist a mechanism which selects only certain oscillators (particles or information); here we choose the external environment of the system to perturb the system such that $g(\chi)$ has a non-stationary, non-zero time-dependent mean that represents the extent to which the system is perturbed. In our model, for a pseudo-random length of time the noise term in each Kuramoto oscillator will be perturbed to a 'new' frequency randomly selected from $g(\omega)$, after 
which the oscillator is returned to its original state; the process will then repeat after a pseudo-random length of time. This process represents the response of neuronal networks to 'pulses' of incoming information, after which the network returns to the quiescent state until another pulse is received.

\section{A quantitative method for comparing models and real data}

It is essential to be able to estimate quantitatively how well the model agrees with real time-series data. To achieve this, we make use of a new parameter $\kappa$ [12], providing a measure of the degree and nature of the coherence or synchronization in the system. Consider $N$ time-series signals of the form

$$
s(t)=\frac{1}{N} \sum_{n=1}^{N} s_{n}(t)
$$

where $s_{n}(t) \in \mathbb{R}$ is the $n^{t h}$ signal. The Hilbert transform gives an analytic signal:

$$
r(t)=\frac{1}{N} \sum_{n=1}^{N} a_{n}(t) e^{i \phi_{n}(t)}
$$

with $a_{n}(t) \in \mathbb{R}^{+}$. The first moment of the magnitude of Eq. (5) and the first moment of the square of Eq. (5) may be expressed respectively as:

$$
\begin{aligned}
& M_{|r|}^{1}=\frac{1}{N} \sqrt{N \mathcal{J}+\left(N^{2}-N\right) \mathcal{J K}}, \\
& M_{r^{2}}^{1}=\frac{1}{N^{2}}\left\{N \mathcal{L}+\left(N^{2}-N\right) \mathcal{L} \mathcal{M}\right\},
\end{aligned}
$$

with

$$
\begin{array}{ll}
\mathcal{J}=\left|\int_{0}^{\infty} \alpha P(\alpha) d \alpha\right|^{2}, & \mathcal{K}=\left|\int_{-\pi}^{\pi} e^{i \theta} P(\theta) d \theta\right|^{2}, \\
\mathcal{L}=\int_{0}^{\infty} \alpha^{2} P(\alpha) d \alpha, & \mathcal{M}=\int_{-\pi}^{\pi} e^{i 2 \theta} P(\theta) d \theta,
\end{array}
$$

where the random variables $\alpha$ and $\theta$ have stochastic distributions $P(\alpha)$ and $P(\theta)$ respectively. The quantity $\kappa$ is defined

$$
\begin{aligned}
\kappa & =\frac{M_{|r|}^{2}-\left(M_{|r|}^{1}\right)^{2}}{M_{r^{2}}^{1}} \\
& =\frac{\operatorname{variance}(|r(t)|)}{\operatorname{mean}\left(r^{2}(t)\right)}
\end{aligned}
$$

where $M_{|r|}^{2}=M_{r^{2}}^{1}$. Hence a single time-series signal (e.g. from an EEG channel) can be Hilbert-transformed, from which $\kappa$ can be found with Eq. (9b). This is directly comparable with $\kappa$ calculated from a model using Eq. (4-9a).

The rescaled variance of the mean field, $\kappa$, provides a useful means of distinguishing between the different states of a coupled ensemble, regardless of the total 
strength of the mean field. As oscillators synchronize into a steady state, the amplitude of the mean field oscillations stabilizes and the variance falls. If all $s_{n}(t)$ signals are perfectly synchronized then $\kappa \rightarrow 0$ (or equivalently the mean-field amplitude $\Psi(t) \rightarrow 1$ ). Conversely, if the ensemble is de-synchronized and each signal $s_{n}(t)$ is composed of Gaussian white noise with zero mean and width $\sigma$, then both the real and imaginary parts of Eq. (5) belong to independent Gaussian distributions. It follows that $a_{n}(t)$ belongs to a Rayleigh distribution and $\phi_{n}(t)$ to a uniform distribution. Therefore when $P(\alpha)$ is a normalized Rayleigh distribution (with $\alpha \geqslant 0, \sigma>0$ ) and $P(\theta)$ is a normalized uniform distribution, the maximally de-synchronized noisy case has,

$$
\kappa=1-\frac{\pi}{4} \simeq 0.215
$$

with $M_{|r|}^{1}=\sigma \sqrt{\frac{\pi}{2 N}}, M_{|r|}^{2}=M_{r^{2}}^{1}=\sigma^{2} \frac{2}{N}$. Consequently for the Kuramoto model, where $a_{n}(t)=1 \forall n, t$, the partially synchronized case yields $0<\kappa<0.215$ which is analogous to $1>\Psi(t)>0$ as defined in Eq. (2) indicating the degree of partial synchronization.

Consider a multi-stable system, in the simplest case making transitions between two states. Define $\varepsilon$ to be the ratio of the mean-field amplitude of the lesssynchronized state to the more-synchronized state, and similarly define $\tau$ to be the portion of time spent in the more-synchronized state divided by the portion of time in the less-synchronized state. An estimate of $\kappa$ is then:

$$
\kappa \approx 1-\frac{[\tau+(1-\tau) \varepsilon]^{2}}{\tau+(1-\tau) \varepsilon^{2}} .
$$

For a constant and high mean-field amplitude $(\Psi(t) \approx 1 \forall t), \varepsilon=1$ so that, as required, $\kappa=0$. Crucially, however, these intermittent cases can result in $\kappa>0.215$. Elsewhere [12] we have used the classic Kuramoto Eq. (1) but with a time-dependent coupling $K(t)$. This parameter was periodically pulsed between two values in order to switch the whole ensemble between the synchronized and desynchronized states. From this we concluded that the smaller the proportion of time spent in the synchronized state, the higher $\kappa$ becomes - tending towards unity for small $\tau$; in this instance if $\tau \lesssim 0.78$ then $\kappa>0.215$

\section{Analysis of brainwaves using EEG}

EEG signals measured on the forehead are the superposition of post-synaptic potentials resulting from the synchronous activity of tens of thousands of neurons in the cerebral cortex. The brain maintains a balance between having either too much, or insufficient, synchronization between neurons; either state will potentially impair information transmission or processing (for example too much synchrony is seen in epilepsy and Parkinson's disease $[13,14])$. We interpret the mean electric field measured by EEG, and the mean field of an ensemble of Kuramoto oscillators, as being analogous. In order to guide our modeling, $\kappa$ will be applied to an EEG time-series signal to gauge the nature of the synchronization. 
(a)

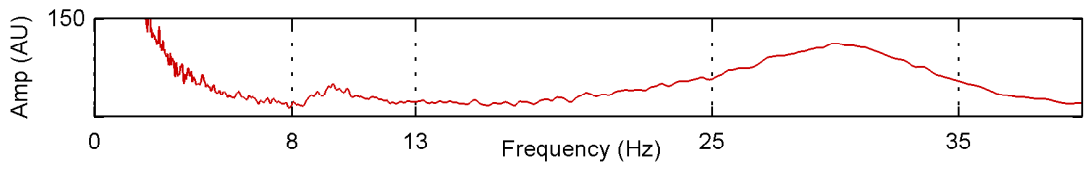

(b)

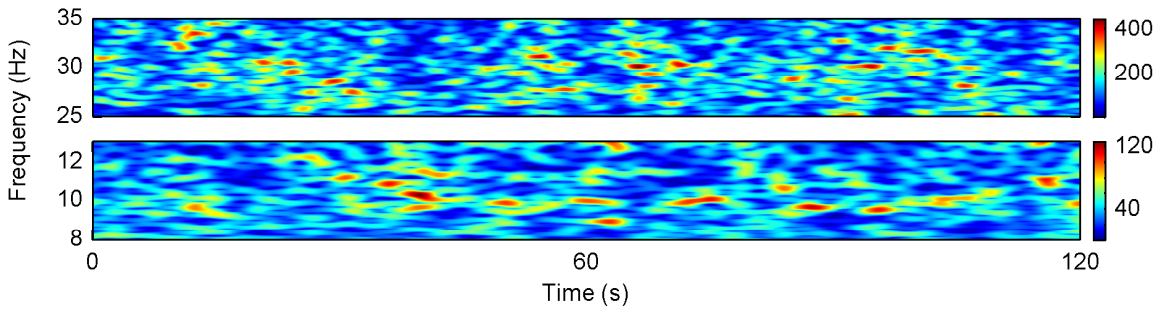

Fig. 1. (Color on-line) Wavelet transform analysis of the EEG signal from a typical [16-19] awake, healthy subject. (a) shows the time-averaged wavelet amplitude, with alpha and gamma peaks at $9.9 \mathrm{~Hz}$ and $29.5 \mathrm{~Hz}$ respectively. Vertical dashed lines indicate the alpha band $(8-13 \mathrm{~Hz})$ and the portion of the gamma $(25-35 \mathrm{~Hz})$ centered about the peak amplitude. The full wavelet transforms (b) and (c) show the time-frequency characteristics of the signal for the alpha (c) and gamma (b) bands. The intensity of activity at each frequency is indicated by the color scale.

We use a single EEG time-series signal taken from the EU FP6 BRACCIA project data-set. The measurements were recorded from a healthy, relaxed, awake adult with eyes closed; extensive data analysis of the BRACCIA data set (not shown here) demonstrates that our selected EEG signal is typical. It is free of movement artifacts. BIS ${ }^{\mathrm{TM}}$ Quatro (two channel) electrodes were located on the forehead, and 20 minutes of data were sampled at $1200 \mathrm{~Hz}$. Fig. 1 shows the frequency spectrum, with obvious peaks in the alpha $(8-13 \mathrm{~Hz})$ and gamma $(>20 \mathrm{~Hz})$ bands at $9.9 \mathrm{~Hz}$ and $29.5 \mathrm{~Hz}$, respectively (we follow Steriade, 2006 [15] and coalesce traditional beta $(13-30 \mathrm{~Hz})$ and gamma $(>30 \mathrm{~Hz})$ into a single gamma band). Viewing these signals in the time-frequency domain (Fig. 1(b) and 1(c)) reveals the occurrence of large-amplitude (red) epochs, indicating high synchronization: if the superposition of membrane potentials adds constructively, these epochs will indicate periods of time when the there is more synchrony. The greater intermittency of highsynchronization epochs in the gamma band corresponds to the attribution of gamma as reflecting neuronal synchrony amongst small, localized ensembles carrying out specific cognitive tasks [16]. On the other hand, alpha activity is considered to reflect the synchronization/desynchronization of more substantial neuronal groups over larger distances, hence facilitating higher-order cognitive tasks (i.e. those of longer duration) [16,17].

To estimate the nature of this synchronization the 20 minute EEG time series signal was Hilbert-transformed, after which Eq. (9b) was used to obtain $\kappa$ over windows of $60 \mathrm{~s}$; from which the mean and standard deviation of $\kappa$ were calculated. Exactly the same procedure was also carried out on a signal composed of Gaussian white noise in order to compare brain dynamics with random processes. 
For the recording from this awake but inactive subject it was found that for the alpha band, $\kappa_{\alpha}=0.235 \pm 0.018$ and $\kappa_{\text {noise }}=0.215 \pm 0.009$; for gamma, $\kappa_{\gamma}=$ $0.227 \pm 0.015$ and $\kappa_{\text {noise }}=0.215 \pm 0.008$. Values calculated from the full signal (without windowing) were very similar. Alpha activity was less intermittent, i.e. had longer periods of synchrony, than gamma activity. This result is consistent with present knowledge of these rhythms, since gamma is known to be associated with more spatially and temporally localized activity than alpha [16]. The proximity of the $\kappa_{\gamma}$ value to $\kappa_{\text {noise }}$ is reasonable, since the more spatially and temporally localized, and the less synchronous, a signal becomes, the more it resembles noise.

In addition to intermittency, amplitude synchronization may also result in $\kappa>$ 0.215 but this is unlikely in a highly coupled system; so our EEG $\kappa$ values indicate either amplitude synchronization (possibly intermittent) and/or purely intermittent phase coherence. Moreover Fig. 1(b) and 1(c) confirm the intermittent nature of increased synchrony.

\section{Modeling brain dynamics as an open system}

In humans the relatively thick cortex is directly responsible for the EEG voltages, but it is also strongly influenced by other brain regions, notably the thalamus. To create a useful model of brain dynamics it is necessary, at the very least, to consider the brain as two separate but connected regions: the thalamus and the cortex. Simplistically the thalamus is responsible for transmitting, filtering and generally organizing information flow, while the cortex is responsible for all the information processing, including cognitive functions. In our model we consider a half (positive frequencies only) unimodal Cauchy distribution $g(\omega)$ of background low frequencies; note that its mean would be zero if both halves were included. Additionally, we have two active symmetric unimodal Cauchy distributions $g(\chi)$ of higher frequencies (notionally emanating from the cortex). It is the higher frequencies introduced through $\chi_{p}(t)$ that are responsible for the intermittency. In a phase dynamics picture, intermittency may be thought of as arising from frequency modulation i.e. in the absence of amplitude modulation.

There are many studies reporting intrinsic rhythms associated with particular local groups of neurons, e.g. clock-like delta in some thalamic neurons [20-22], but here we take a more global view. The frequency spectra of EEG time-series signals show that the awake brain is primarily concerned with processing incoming information and is perturbed away from its intrinsic resting state by external demands. Consequently the awake EEG (Fig. 1) contains significant power at higher frequencies (predominantly 20-50 Hz) compared with an asleep EEG (not shown here). However, in the awake but inactive brain with eyes closed, a rhythm of $\sim 10 \mathrm{~Hz}$ is also clearly visible. This alpha rhythm is associated with active inhibition of cortical areas [17] and as such is a less intermittent characteristic than activity in the gamma band. We consider first the most prominent features in Fig. 1 for which we set the mean of $g(\chi)$ to be 30 and $10 \mathrm{~Hz}$, respectively, for the gamma and alpha 


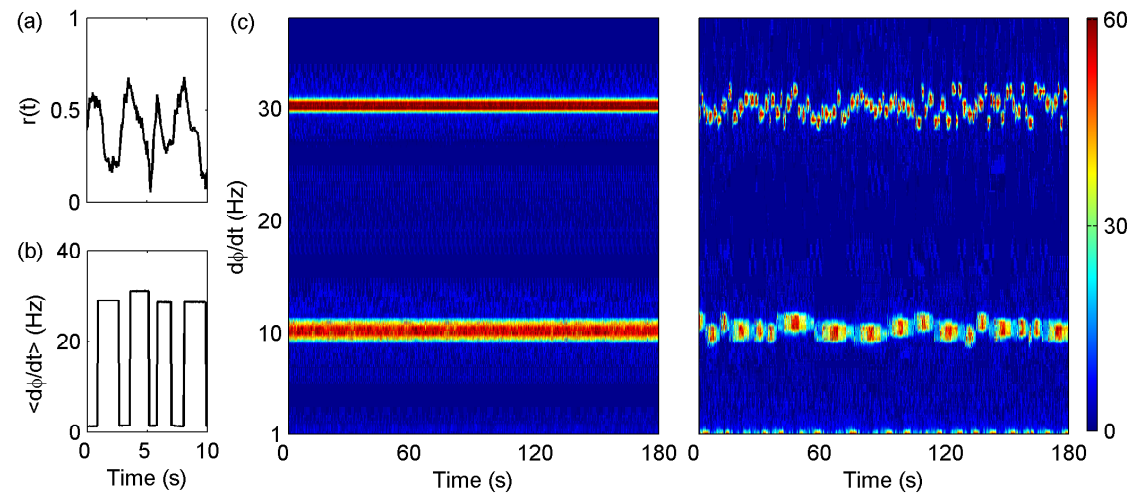

Fig. 2. (Color) For a subset of gamma oscillators the mean field (a) and averaged $\dot{\phi}(t)$ (b) are depicted. The mean phase $\left\langle\dot{\phi}_{p}(t)\right\rangle$ is the sum of each oscillator in the subset divided by the number of oscillators in the subset. The nature of the noise introduced by $g(\chi)$ is shown through $\left\langle\dot{\phi}_{p}(t)\right\rangle$. The mean field (a) is subject to the effect of the other oscillators in the network so is directly influenced by the noise and perturbed by the rest of the network. In (c), values of $\dot{\phi}(t)$ from the model are plotted as a histogram, with the data separated into frequency bins over a range of 1-40 Hz. These histograms are comparable to the wavelet transforms in Fig. 1, with the same color scale indicating high (red) to low (blue) synchrony. In (c), the left panel is for stationary $g(\chi)$, i.e. the closed Kuramoto system Eq. (1) and on the right are results for our open model Eq. (3) showing intermittency. The simulation uses a fourth order Runge-Kutta algorithm with $N=500$ oscillators; of these $20 \%$ are unperturbed and of the remaining $80 \%$ half belong to the alpha band (centered around $10 \mathrm{~Hz}$ ) and half to the gamma band (centered around $30 \mathrm{~Hz}$ ). The coupling is set at $K=5 \mathrm{rad} / \mathrm{s}$.

sub-ensembles, with a half-width of $0.1 \mathrm{~Hz}$ in each case.

Initially the eigenfrequencies for all $N$ oscillators are selected from $g(\omega)$ and $20 \%$ of all oscillators remain as a background and never have their eigenfrequencies perturbed, nominally representing cortical neurons that are strongly influenced by thalamic input. In order to create the intermittency, $\mathcal{R}(t)$ determines the period of time during which the remaining $80 \%$ of oscillators are temporarily perturbed away from their eigenfrequencies $\left(\chi_{p}(t) \neq 0\right)$, before being returned again $\left(\chi_{p}(t)=0\right)$. The alpha and gamma bands each contain $40 \%$ of the oscillators. The perturbed frequency of the $p^{t h}$ oscillator is selected from $g(\chi)$ and applied through the $\chi_{p}(t)$ term; $\chi_{p}=0$ represents neuronal groups with little or no excitatory input and conversely $\chi_{p} \neq 0$ represents excitatory processes. At the end of each interval $\mathcal{R}_{a}(t)$ a new $\mathcal{R}_{a+1}(t)$ is selected using $\mathcal{R}_{a+1}(t)=\Gamma+2 \Gamma(\eta(t)-1 / 2)$ where $\Gamma$ is a constant background rate (different for the two bands) and $\eta(t)$ is number drawn from a uniform distribution with zero mean; in the gamma sub-ensemble by $\Gamma=20 \times 0.05 \mathrm{~s}$ and in the alpha sub-ensemble by $\Gamma=180 \times 0.05 \mathrm{~s}$. The $\Gamma$ for each band is guided by examination of the EEG data (Fig. 1(b) and 1(c)) in order to ensure that approximately the right number of synchronized events occur. The term $\chi_{p}(t) \neq 0$ over an interval of $\frac{1}{2} \mathcal{R}_{a}(t)$ in both sub-ensembles. At the beginning of every time length $\mathcal{R}_{a}(t)$ the mean of $g(\chi)$ is adjusted by $2 \pi(30+3(\xi(t)-0.5))$ 
in the gamma sub-ensemble and $2 \pi(10+2(\xi(t)-0.5))$ in the alpha sub-ensemble, where $\xi(t)$ is a number drawn from a uniform distribution over the interval $[0,1]$. In summary the mean frequency of $g(\chi)$ is adjusted at the beginning of the time interval $\mathcal{R}_{a}(t)$. Afterwards its value is updated to $\mathcal{R}_{a+1}(t)$; and then the whole process repeats.

The left plot in Fig. 2(c) shows model results for the case where $g(\chi)$ is stationary, i.e. the closed Kuramoto system (1): the 10 and $30 \mathrm{~Hz}$ bands do not exhibit time-variability, in stark contrast to the left plot in Fig. 2(c) showing the model results for our open system. In the closed case the alpha and gamma sub-ensembles have $\kappa \simeq 0.19 \pm 0.02$ which is consistent with the stationary partially-coupled case; in the open case the alpha sub-ensemble has $\kappa=0.24 \pm 0.02$ and gamma $\kappa=0.22 \pm 0.01$ (standard deviation obtained with a $20 \mathrm{~min}$ signal split into $60 \mathrm{~s}$ windows). These values are very similar to the EEG results where $\kappa_{\alpha}=0.23 \pm 0.02$, $\kappa_{\gamma}=0.23 \pm 0.01$. With a phase dynamics approach, intermittency is evidently necessary to obtain realistic $\kappa$ values.

\section{Conclusion}

We have shown that, if extended to include a noise term to simulate the effect of the environment, an ensemble of Kuramoto oscillators can provide a satisfactory description of the intermittent and episodic character of the synchronization seen in EEG signals. We used the parameter $\kappa[12]$ to quantify the degree and type of synchronization, enabling the model to be compared with real data. When the epochs of synchronization in the model occur on similar time-scales to those seen in the EEG time-series, both the model and the EEG data yield similar values of $\kappa \sim 0.23$. This is to be compared with the classic Kuramoto model which, in the absence of intermittent synchronization, with or without white noise, exhibits $\kappa$ values in the range $0<\kappa<0.215$, where 0.215 represents the white noise limit.

Intermittency is an integral aspect of brain dynamics and should be taken into account when modeling. Because much of the information (or matter or energy) flow is unpredictable from the perspective of a cell receiving it, this intermittency must be incorporated within the model by the introduction of a term that is subject to some degree of noise (randomness). We have suggested one way of doing so, but there are of course other possible approaches that can be explored in the future. Given that most biological systems are open in the sense that both matter and information pass continuously in and out, and that many of these systems are selfsynchronizing, we believe that our approach will be widely applicable.

\section{Acknowledgements}

The authors are grateful to the Wellcome Trust for financial support of this research and to the EU FP6 BRACCIA project consortium for the provision of EEG data. 


\section{References}

[1] S. H. Strogatz, "From Kuramoto to Crawford: exploring the onset of synchronization in populations of coupled oscillators", Physica D 143 (2000) 1-20.

[2] A. Pikovsky, M. Rosenblum and J. Kurths, Synchronization: A Universal Concept in Nonlinear Sciences (Cambridge University Press, 2003).

[3] E. M. Izhikevich, "Which model to use for cortical spiking neurons?", IEEE Trans. Neural. Nets. 15 (2004) 1063-1070.

[4] H. R. Wilson and J. D. Cowan, "Excitatory and inhibitory interactions in localized populations of model neurons", Biophys. J. 12 (1972) 1-24.

[5] D. T. J. Liley, P. J. Cadusch and J. J. Wright, "A continuous theory of electro-cortical activity", Neurocomp. 26-27 (1999) 795-800.

[6] V. K. Jirsa and H. H Haken, "Field theory of electromagnetic brain activity", Phys. Rev. Lett. 77 (1996) 960-963.

[7] C. Laing and G. J. Lord, Stochastic Methods in Neuroscience (OUP, Oxford, 2009).

[8] M. Breakspear, S. Heitmann and A. Daffertshofer, "Generative models of cortical oscillations: neurobiological implications of the Kuramoto model", Frontiers in Human Neurosci. 4 (2010) 1-14.

[9] J. H. Sheeba, A. Stefanovska and P. V. E. McClintock, "Neuronal synchrony during anesthesia: a thalamocortical model", Biophys. J. 95 (2008) 2722-2727.

[10] Y. Maistrenko, B. Lysyansky, C. Hauptmann, O. Burylko and P. Tass, "Multistability in the Kuramoto model with synaptic plasticity", Phys. Rev. E 75 (2007) 1-8.

[11] C. Koch and S. Green, "How does consciousness happen?", Scientific American 297 (2007) $76-83$.

[12] L. W. Sheppard, A. C. Hale, S. Petkoski, A. Stefanovska and P. V. E. McClintock, "More random than random: the mean field of interacting oscillator ensembles", to be published.

[13] J. R. Hughes, "Gamma, fast, and ultrafast waves of the brain: Their relationships with epilepsy and behaviour", Epilepsy and Behaviour 13 (2008) 25-31.

[14] R. Levy, W. D. Hutchinon, A. M. Lozano and J. O. Dostrovsky, "High-frequency synchronization of neuronal activity in the subthalamic nucleus of parkinsonian patients with limb tremor", J. Neurosci. 20 (2000) 7766-7775.

[15] M. Steriade, "Grouping of brain rhythms in corticothalamic systems", Neurosci. 137 (2006) 1087-1106.

[16] A. Stein and J. Sarnthein, "Different frequencies for different scales of cortical integration: from local gamma to long range alpha/theta synchronization", J. Neurosci. 38 (2000) 301-313.

[17] W. Klimesch, P. Sauseng and S. Hanslmayr, "EEG alpha oscillations: The inhibitiontiming hypothesis", Brain Res. Rev. 53 (2007) 63-88.

[18] E. Niedermeyer, "Alpha rhythms as physiological and abnormal phenomena", International journal of psychophysiology 26 (1997) 31-49.

[19] A. C. N. Chen, W. Feng, H. Zhao, Y. Yin and P. Wang, "EEG default mode network in the human brain: Spectral regional field powers", NeuroImage 41 (2008) 561-574.

[20] M. A. Long, C. E. Landisman and B. W. Connors, "Small clusters of electrically coupled neurons generate synchronous rhythms in the thalamic reticular nucleus", $J$. Neurosci. 24 (2004) 341-349.

[21] F. Amzica and M. Steriade, "Electrophysiological correlates of sleep delta waves", Electroenceph. clin. Neurophysio. 107 (1998) 69-83.

[22] M. Steriade, I. Timofeev, N. Dürmüller and F. Grenier, "Dynamic properties of corticothalamic neurons and local cortical interneurons generating fast rhythmic (3040 hz) spike bursts", J. Neurophysiol. 79 (1998) 483-490. 